\section{Università $\underbrace{1}_{0,0 B=58}$ \\ INTRODUCTION \\ Synthetic opioids are a large class of new psychoactive substances (NPS) mostly consisting of analogs of fentanyl (Fen) that have caused numerous overdose and fatalities worldwide. In 2016 the European Monitoring Centre for Drugs and Drug Addiction (EMCDDA) has reported a total of 130 deaths caused by a new psychoactive substance called "Acrylofentanyl" in United States and Europe. Acrylofentanyl (AcrylF) was spreading online as a research chemical and was mostly sold in a powder or nasa spray forms. It is a potent NPS that is being used and abused causing more than 21 intoxications and 47 deaths in Europe within a relatively short time period. The aim of the present study was to compare the acute effects evoked by AcrylF, Fen and morphine (mor) on motor, sensorimotor and cardio-respiratory changes in mice. To better understand the pharmacological action of AcrylF, we used naloxone as opioid receptor antagonist.}

\title{
METHODS
}

Adult male ICR (CD-1) mice, weighing from 25 to 30 grams, were used for this experiment We used for this study the range of doses $(0.01-15 \mathrm{mg} / \mathrm{kg})$, and the three compounds were administered by intraperitoneal injection at a volume of $4 \mu \mathrm{l} / \mathrm{g}$. The opioid receptol antagonist naloxone $(6 \mathrm{mg} / \mathrm{kg}$, i.p.) was administered 15 minutes before AcrylF, Fen an morphine injections. The effect of AcrylFen, Fen and mor was investigated using a battery of behavioral tests widely used in studies of "safety-pharmacology" for the preclinical characterization of new molecules in rodents. All experiments were performec between 8:30 AM to 2:00 PM. Experiments were conducted in blind by trained observers working together in pairs. The behavior of mice was videotaped and analyzed off-line by a different trained operator that gives test scores. Spontaneous locomotor activity was measured in four mice for each experiment where motor activity was monitored for 240 min by using the ANY-maze video-tracking system. Cardio-respiratory parameters (heart rate, breath rate, oxygen saturation and pulse distention) were measured through the software MouseOx Plus in freely moving mice, monitored by a sensor collar applied around their neck and data is recorded for 5 hours

\section{TAIL PINCH}

Fig 3: Effect of the systemic administration of mor. Fen and AcrylF $(0.01-15 \mathrm{mg} / \mathrm{kg}$ i.p.) on the tail pinch test of the mouse. Interaction of effective dose of the three compounds $(6 \mathrm{mg} / \mathrm{kg})$ with the opioid receptor antagonist naloxone $(6$ $\mathrm{mg} / \mathrm{kg}$, i.p.; panel B, D and F, respectively) and he comparison of their maximal effects panel $G$. Data are expressed as percentage of maximum effect and represent the mean \pm SEM of 8 determinations for each treatment. Statistical analysis was performed by two-way ANOVA followed by the Bonferroni's test for multiple comparisons. $\quad{ }^{*} p<0.05, \quad{ }^{* *} p<0.01, \quad{ }^{* * *} p<0.001$ versus vehicle

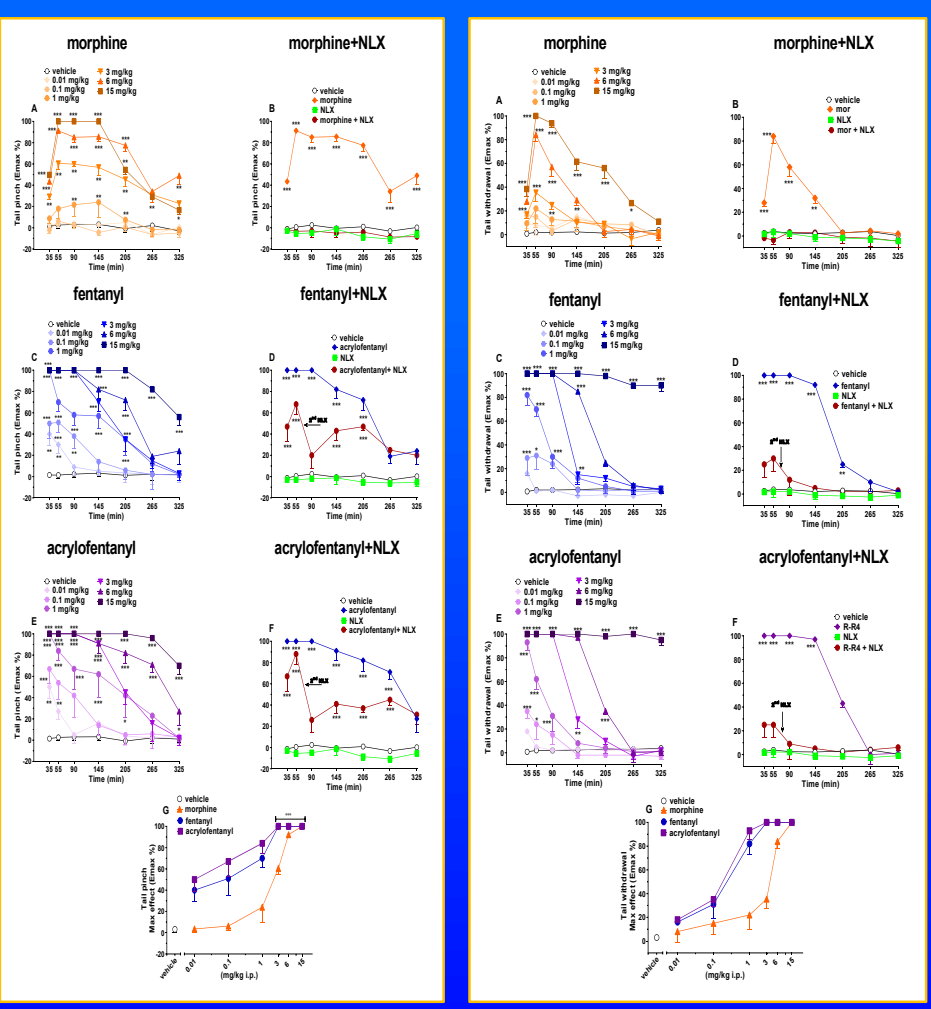

\section{RESULTS}

AcrylF, Fen and mor (0.01- $15 \mathrm{mg} / \mathrm{kg}$ i.p.), dose-dependently inhibited visual object and visual placing responses in mice. In particular, the highest dose tested (15 mg/kg i.p.) of both fentanyl drugs inhibited totally $(100 \%)$ the visual responses in both tests after 10 min of injection. AcrylF induced a long-lasting analgesic effect similar to Fen but more effective at low doses than mor. The maximal antinociceptive effect in mechanical and thermal test was reached after $35 \mathrm{~min}$ of injection of both fentanyl drugs. AcrylF and Fen (15 mg/kg i.p.) facilitated spontaneous motor activity, while mor at the same dose was ineffective. All the three compounds reduced heart and breath rates. AcrylF at the dose of $6 \mathrm{mg} / \mathrm{kg}$ induced a reduction of $35 \%$ of the heart rate and $30 \%$ of breath rate after $10 \mathrm{~min}$ of injection. The dose of $6 \mathrm{mg} / \mathrm{kg}$ of naloxone was partially effective to prevent the impairment caused by AcrylF and Fen while the same dose prevented all the effects conducted by morphine.

\begin{tabular}{|c|}
\hline 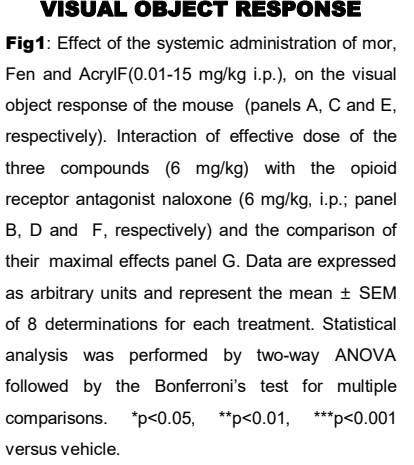 \\
\hline
\end{tabular}

\section{VISUAL PLACING RESPONSE}

Fig2: Effect of the systemic administration of mor, Fen and AcrylF (0.01-15 mg/kg i.p.), on the visual placing response of the mouse (panels A, $C$ and $E$, respectively), Interaction of effective dose of the three compounds $(6 \mathrm{mg} / \mathrm{kg})$ with the opioid receptor antagonist naloxone $(6 \mathrm{mg} / \mathrm{kg}$, i.p.; panel $B, D$ and $F$, respectively) and the comparison of their maximal effects panel $G$. Data are expressed as arbitrary units and represent the mean \pm SEM of 8 determinations for each treatment. Statistical analysis was performed by two-way ANOVA followed by the Bonferroni's test for multiple comparisons. ${ }^{*} p<0.05,{ }^{, *} p<0.01,{ }^{* * *} p<0.001$ versus vehicle.

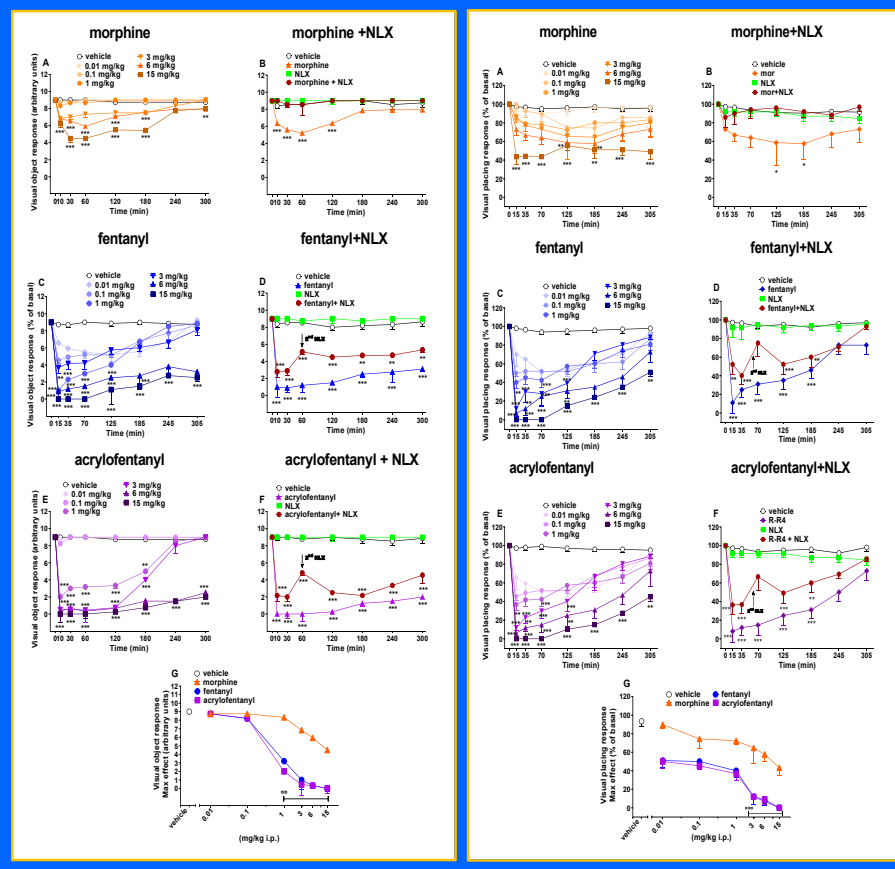

\section{SPONTANEOUS LOCOMOTION}

Fig 4: Effect of the systemic administration of mor (panel A), Fen (panel B) and AcrylF (panel C), (0.01-15 $\mathrm{mg} / \mathrm{kg}$ i.p.) on the total distance travelled test of the mouse. Data are expressed as meters travelled and represent the mean \pm SEM of 10 determinations for each treatment. Statistical analysis was performed by two-way ANOVA followed by the Bonferroni's test for multiple comparisons. ${ }^{*} p<0.05,{ }^{* *} p<0.01,{ }^{* * *} p<0.001$ versus vehicle.

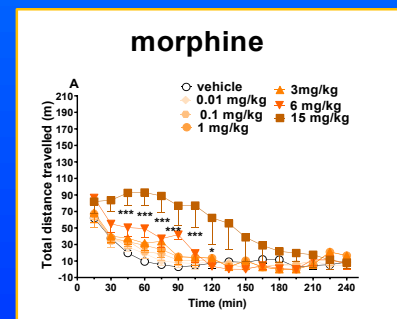

acrylofentanyl

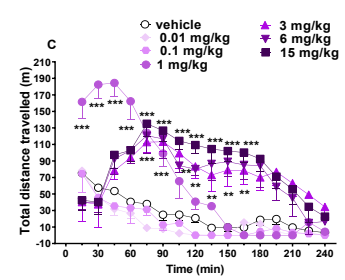




\section{HEART RATE}

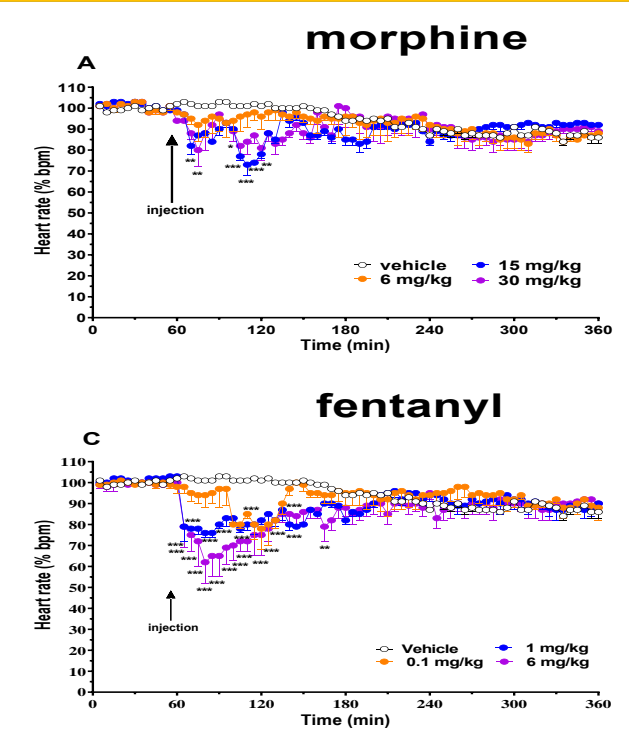

acrylofentanyl

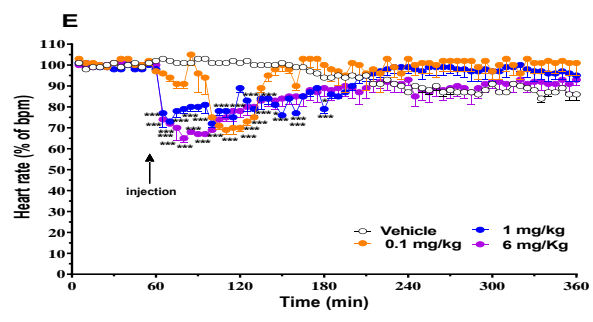

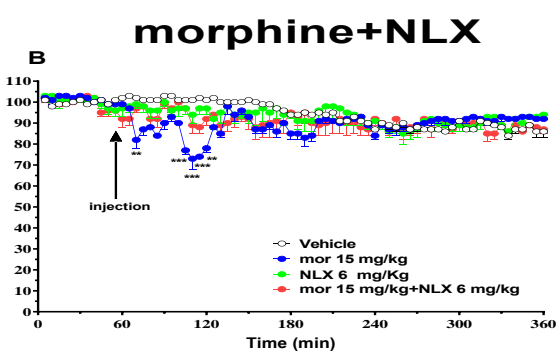

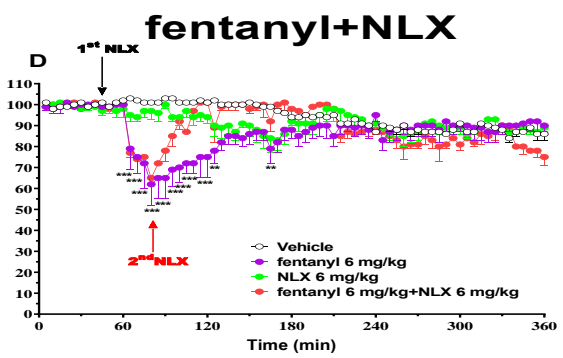

acrylofentanyI+NLX

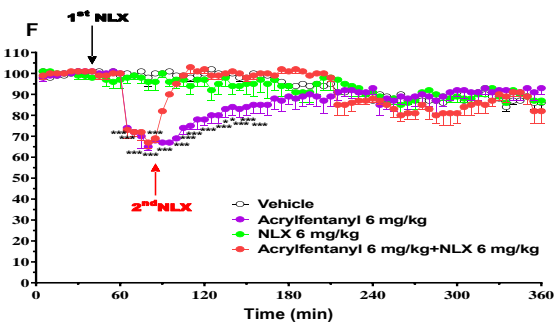

\section{BREATH RATE}

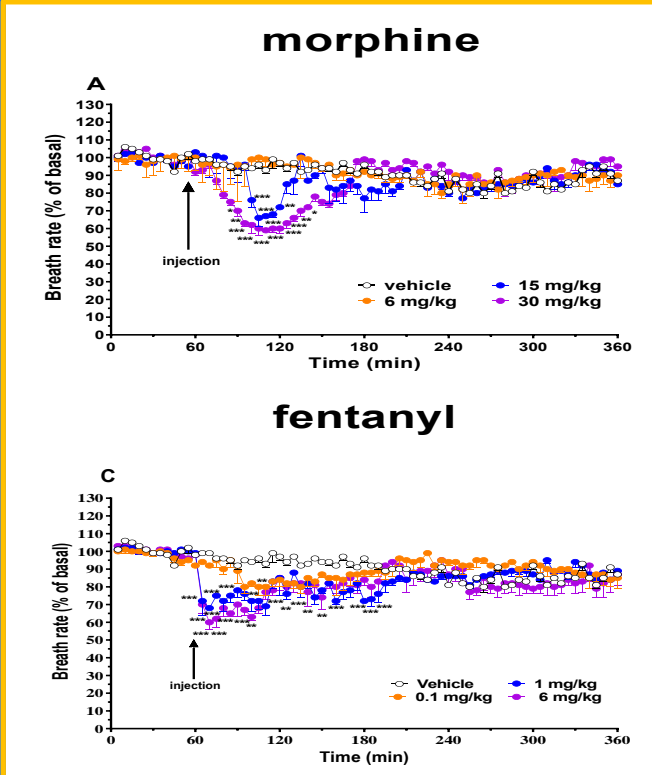

\section{acrylofentanyl}

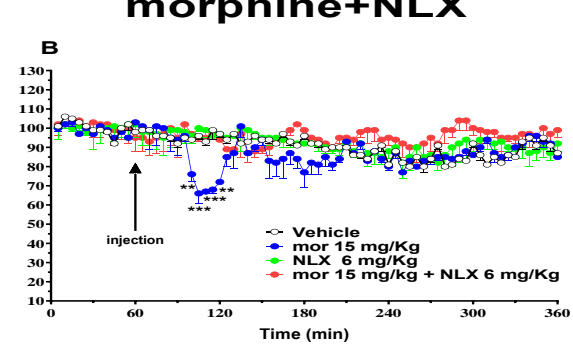

\section{fentanyl +NLX}

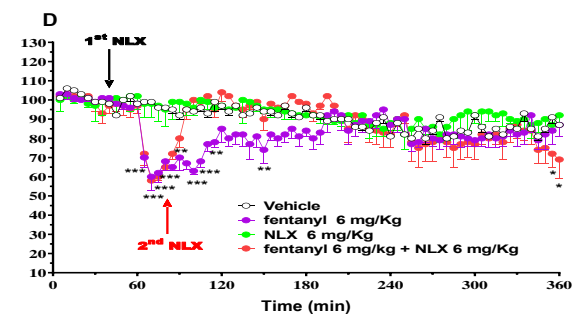

\section{acrylofentanyl +NLX}
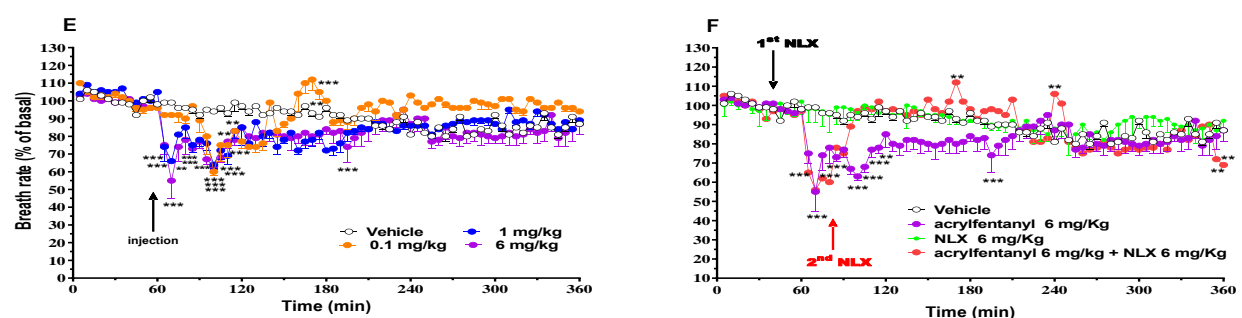

\section{CONCLUSION}

The present study demonstrates that acute administration of AcrylF and Fen markedly inhibits visual object and visual placing responses in mice, facilitates spontaneous locomotion and impairs cardio-respiratory parameters with a persistent respiratory depression in mice. Pre-treatment with naloxone was partially effective to block the effects of AcrylF and Fen while the same dose was effective to block all the impairments induced by morphine even at the dose of 15 $\mathrm{mg} / \mathrm{kg}$. These findings support the harmful health risks of fentanyl and its analogues and their high capacity to induce fatality

\section{REFERENCES}

Ossato et al., (2015) Neuroscience; 300:174-88

Bilel et al.,(2019) Neurotoxicology;11;76:17-32

Bilel et al .,(2019) Frontiers Neuroscience; 01163

\section{ACKNOWLEDGEMENTS}

This research has been funded by the Drug Policies Department Presidency of the Council of Ministers, Italy (project:"Effects of NPS: development of a multicentre research for the information enhancement of the Early Warning System" to Matteo Marti). 